\title{
Volume estimates for equiangular hyperbolic Coxeter polyhedra
}

\author{
CHRISTOPHER K ATKINSON
}

\begin{abstract}
An equiangular hyperbolic Coxeter polyhedron is a hyperbolic polyhedron where all dihedral angles are equal to $\pi / n$ for some fixed $n \in \mathbb{Z}, n \geq 2$. It is a consequence of Andreev's theorem that either $n=3$ and the polyhedron has all ideal vertices or that $n=2$. Volume estimates are given for all equiangular hyperbolic Coxeter polyhedra.
\end{abstract}

57M50; 30F40

\section{Introduction}

An orientable 3 -orbifold, $\mathcal{Q}$, is determined by an underlying 3-manifold, $X_{\mathcal{Q}}$ and a trivalent graph, $\Sigma_{\mathcal{Q}}$, labeled by integers. If $\mathcal{Q}$ carries a hyperbolic structure then it is unique by Mostow rigidity, so the hyperbolic volume of $\mathcal{Q}$ is an invariant of $\mathcal{Q}$. Therefore, for hyperbolic orbifolds with a fixed underlying manifold, the volume is a function of the labeled graph $\Sigma$. In this paper, methods for estimating the volume of orbifolds of a restricted type in terms of this labeled graph will be described.

The orbifolds studied in this paper are quotients of $\mathbb{H}^{3}$ by reflection groups generated by reflections in hyperbolic Coxeter polyhedra. A Coxeter polyhedron is one where each dihedral angle is of the form $\pi / n$ for some $n \in \mathbb{Z}, n \geq 2$. Given a hyperbolic Coxeter polyhedron $\mathcal{P}$, consider the group generated by reflections through the geodesic planes determined by its faces, $\Gamma(\mathcal{P})$. Then $\Gamma(\mathcal{P})$ is a Kleinian group which acts on $\mathbb{H}^{3}$ with fundamental domain $\mathcal{P}$. The quotient, $\mathcal{O}=\mathbb{H}^{3} / \Gamma(\mathcal{P})$, is a nonorientable orbifold with singular locus $\mathcal{P}^{(2)}$, the 2 -skeleton of $\mathcal{P}$. One may think of obtaining $\mathcal{O}$ by "mirroring" the faces of $\mathcal{P}$. It is a consequence of Andreev's theorem that any equiangular hyperbolic Coxeter polyhedron has either all dihedral angles equal to $\pi / 3$ and is ideal or has all dihedral angles equal to $\pi / 2[5 ; 6]$. This paper gives two-sided combinatorial volume estimates for all equiangular hyperbolic Coxeter polyhedra. Only polyhedra with finite volume will be considered.

Lackenby gave volume estimates for hyperbolic alternating link complements in [14] in terms of the twist number of the link. His work was part of what led to this investigation of how geometric data arises from associated combinatorial data. Some 
of the techniques used in this paper follow methods used by Lackenby. The lower bound given by Lackenby was improved by Agol, Storm and Thurston in [3]. In his thesis [13], Inoue has identified the two smallest-volume, compact, right-angled hyperbolic polyhedra. He also gave a method to order such polyhedra based on a decomposition into Löbell polyhedra, provided that the volume of any given rightangled polyhedron can be calculated exactly.

The results of this paper can be used to list all equiangular hyperbolic polyhedra with volume not exceeding some fixed value. A sample application of this is to classify all arithmetic Kleinian maximal reflection groups. Agol has shown in [2] that the number of such groups is finite up to conjugacy. Given a maximal reflection group $\Gamma$ generated by reflections in a polyhedron $\mathcal{P}$, he gives an upper bound, independent of $\Gamma$, for the volume of $\mathcal{P} / \Theta$ where $\Theta$ is the group of symmetries of $\mathcal{P}$ which are not reflections. One could therefore attempt to classify such groups using the results of this paper by writing down a list of all polyhedra of sufficiently small volume and checking arithmeticity for those for which the quotient by additional symmetries has small enough volume.

Acknowledgments The author would like to thank his thesis advisor, Ian Agol, for his excellent guidance and the referee for many valuable comments. The author was partially supported by NSF grant DMS-0504975.

\section{Summary of results}

The results of the paper are outlined in this section. Theorems 2.2, 2.3 and 2.4 concern the volumes of right-angled hyperbolic polyhedra. Theorem 2.6 concerns hyperbolic polyhedra with all angles $\pi / 3$. Before stating any results, some terminology will be introduced.

An abstract polyhedron is a cell complex on $S^{2}$ which can be realized by a convex Euclidean polyhedron. A theorem of Steinitz says that realizability as a convex Euclidean polyhedron is equivalent to the 1-skeleton of the cell complex being 3-connected [18]. A graph is 3 -connected if the removal of any 2 vertices along with their incident edges leaves the complement connected. A labeling of an abstract polyhedron $P$ is a map

$$
\Theta: \operatorname{Edges}(P) \rightarrow(0, \pi / 2] \text {. }
$$

For an abstract polyhedron, $P$, and a labeling, $\Theta$, the pair $(P, \Theta)$ is a labeled abstract polyhedron. A labeled abstract polyhedron is said to be realizable as a hyperbolic polyhedron if there exists a hyperbolic polyhedron, $\mathcal{P}$, such that there is a labelpreserving graph isomorphism between $\mathcal{P}^{(1)}$ with edges labeled by dihedral angles 
and $P$ with edges labeled by $\Theta$. A defining plane for a hyperbolic polyhedron $\mathcal{P}$ is a hyperbolic plane $\Pi$ such that $\Pi \cap \mathcal{P}$ is a face of $\mathcal{P}$. A labeling $\Theta$ which is constantly equal to $\pi / n$ is $\pi / n$-equiangular. Suppose $G$ is a graph and $G^{*}$ is its dual graph. A $k$-circuit is a simple closed curve composed of $k$ edges in $G^{*}$. A prismatic $k$-circuit is a $k$-circuit $\gamma$ so that no two edges of $G$ which correspond to edges traversed by $\gamma$ share a vertex. The following theorem is a special case of Andreev's Theorem, which gives necessary and sufficient conditions for a labeled abstract polyhedron to be realizable as a hyperbolic polyhedron.

Theorem 2.1 (Andreev's theorem for $\pi / 2$-equiangular polyhedra) $A \pi / 2$-equiangular labeled abstract polyhedron $(P, \Theta)$ is realizable as a hyperbolic polyhedron, $\mathcal{P}$, if and only if the following conditions hold:

(1) $P$ has at least 6 faces.

(2) $P$ each vertex has degree 3 or degree 4.

(3) For any triple of faces of $P,\left(F_{i}, F_{j}, F_{k}\right)$, such that $F_{i} \cap F_{j}$ and $F_{j} \cap F_{k}$ are edges of $P$ with distinct endpoints, $F_{i} \cap F_{k}=\varnothing$.

(4) $P^{*}$ has no prismatic 4 circuits.

Furthermore, each degree 3 vertex in $P$ corresponds to a finite vertex in $\mathcal{P}$, each degree 4 vertex in $P$ corresponds to an ideal vertex in $\mathcal{P}$, and the realization is unique up to isometry.

The first result gives two-sided volume estimates for ideal, $\pi / 2$-equiangular hyperbolic polyhedra.

Theorem 2.2 If $\mathcal{P}$ is an ideal $\pi / 2$-equiangular polyhedron with $N$ vertices, then

$$
(N-2) \cdot \frac{V_{8}}{4} \leq \operatorname{vol}(\mathcal{P}) \leq(N-4) \cdot \frac{V_{8}}{2},
$$

where $V_{8}$ is the volume of a regular ideal hyperbolic octahedron. Both inequalities are equality when $\mathcal{P}$ is the regular ideal hyperbolic octahedron. There is a sequence of ideal $\pi / 2$-equiangular polyhedra $\mathcal{P}_{i}$ with $N_{i}$ vertices such that $\operatorname{vol}\left(\mathcal{P}_{i}\right) / N_{i}$ approaches $V_{8} / 2$ as $i$ goes to infinity.

The constant $V_{8}$ is the volume of a regular ideal hyperbolic octahedron. In terms of the Lobachevsky function,

$$
\Lambda(\theta)=-\int_{0}^{\theta} \log |2 \sin t| d t
$$


$V_{8}=8 \Lambda(\pi / 4)$. This volume can also be expressed in terms of Catalan's constant, $K$, as $V_{8}=4 K$, where

$$
K=\sum_{n=0}^{\infty} \frac{(-1)^{n}}{(2 n+1)^{2}} .
$$

The value of $V_{8}$ to five decimal places is 3.66386 .

The proof is spread throughout the paper. The lower bound will be shown in Section 3 to be a consequence of the stronger Theorem 3.2, which depends also on information about the number of faces. The upper bound in Theorem 2.2 will be proved in Section 5 and will be shown to be asymptotically sharp in Section 6 .

Using similar techniques, the following theorem giving volume estimates for compact $\pi / 2-$ equiangular polyhedra will be proved:

Theorem 2.3 If $\mathcal{P}$ is a compact $\pi / 2$-equiangular hyperbolic polyhedron with $N$ vertices, then

$$
(N-8) \cdot \frac{V_{8}}{32} \leq \operatorname{vol}(\mathcal{P})<(N-10) \cdot \frac{5 V_{3}}{8},
$$

where $V_{3}$ is the volume of a regular ideal hyperbolic tetrahedron. There is a sequence of compact polyhedra, $\mathcal{P}_{i}$, with $N_{i}$ vertices such that $\operatorname{vol}\left(\mathcal{P}_{i}\right) / N_{i}$ approaches $5 V_{3} / 8$ as $i$ goes to infinity.

In terms of the Lobachevsky function, $V_{3}=2 \Lambda(\pi / 6)$. To five decimal places, $V_{3}$ is 1.01494 .

Combining the methods of Theorem 2.2 and Theorem 2.3, estimates will be given for $\pi / 2$-equiangular polyhedra with both finite and ideal vertices:

Theorem 2.4 If $\mathcal{P}$ is a $\pi / 2$-equiangular hyperbolic polyhedron, $N_{\infty} \geq 1$ ideal vertices and $N_{F}$ finite vertices, then

$$
\frac{8 N_{\infty}+N_{F}-8}{32} \cdot V_{8} \leq \operatorname{vol}(\mathcal{P})<\left(\mathrm{N}_{\infty}-1\right) \cdot \frac{V_{8}}{2}+N_{F} \cdot \frac{5 V_{3}}{8} .
$$

The proofs of the lower bounds in Theorem 2.3 and Theorem 2.4 appear in Section 3. The upper bounds will be proved in Section 5 .

Two-sided volume estimates for $\pi / 3$-equiangular polyhedra are also given. First, the special case of Andreev's Theorem for $\pi / 3$-equiangular polyhedra is stated.

Theorem 2.5 (Andreev's theorem for $\pi / 3$-equiangular polyhedra) $A \pi / 3$-equiangular abstract polyhedron, $(P, \Theta)$, is realizable as a hyperbolic polyhedron, $\mathcal{P}$ if each vertex of $P$ has degree 3 and $P^{*}$ has no prismatic 3-circuits. Furthermore, each vertex of $\mathcal{P}$ is ideal and $\mathcal{P}$ is unique up to isometry. 
The next theorem gives analogous results to that of Theorem 2.2 for ideal $\pi / 3-$ equiangular polyhedra.

Theorem 2.6 If $\mathcal{P}$ is an ideal $\pi / 3$-equiangular polyhedron with $N>4$ vertices, then

$$
N \cdot \frac{V_{3}}{3} \leq \operatorname{vol}(\mathcal{P}) \leq(3 N-14) \cdot \frac{V_{3}}{2},
$$

where $V_{3}$ is the volume of a regular ideal hyperbolic tetrahedron. The upper bound is sharp for the regular ideal hyperbolic cube. There is a sequence of ideal $\pi / 3-$ equiangular polyhedra $\mathcal{P}_{i}$ with $N_{i}$ vertices such that $\operatorname{vol}\left(\mathcal{P}_{i}\right) / N_{i}$ approaches $3 V_{3} / 2$ as $N_{i}$ increases to infinity.

The lower bound in this theorem will be proved in Section 4 by packing horoballs around the vertices. The upper bound will be proved in Section 5 and will be shown to be asymptotically sharp in Section 6 , mirroring the proofs in the $\pi / 2$ case. In a personal communication, Rivin has indicated how to improve the lower bound to $N \cdot\left(3 V_{3} / 8\right)$. His argument will be briefly described at the end of Section 4.

\section{Lower volume bound for ideal $\pi / 2$-equiangular polyhe- dra}

The key result used in proving the lower volume bound in Theorem 2.2 and Theorem 3.2 is a theorem of Miyamoto which says that the volume of a complete hyperbolic 3manifold with totally geodesic boundary is greater than or equal to a constant multiple of the area of the boundary [15]:

Theorem 3.1 (Miyamoto) If $\mathcal{O}$ is a complete hyperbolic 3-orbifold with nonempty totally geodesic boundary, then

$$
\operatorname{vol}(\mathcal{O}) \geq \operatorname{area}(\partial \mathcal{O}) \cdot \frac{V_{8}}{4 \pi}
$$

with equality only if $M$ can be decomposed into regular ideal hyperbolic octahedra.

Miyamoto actually only stated this theorem for manifolds. The orbifold version follows immediately, however. Given a complete hyperbolic 3-orbifold, $\mathcal{O}$, with nonempty totally geodesic boundary, Selberg's lemma implies the existence of an integer $m$ such that an $m$-fold cover of $\mathcal{O}$ is a manifold $M$ [17]. Then since $M$ is a finite cover, $\operatorname{vol}(M)=m \cdot \operatorname{vol}(\mathcal{O})$ and $\operatorname{area}(\partial M)=m \cdot \operatorname{area}(\partial \mathcal{O})$.

Theorem 2.2 is a consequence of the following stronger theorem which also takes into account information about the faces of the polyhedron. Andreev's theorem for 
$\pi / 2$-equiangular polyhedra implies that the 1 -skeleton of an ideal $\pi / 2$-equiangular polyhedron is a four-valent graph on $S^{2}$. The faces, therefore, can be partitioned into a collection of black faces, $\mathcal{B}$, and a collection of white faces, $\mathcal{W}$, so that no two faces of the same color share an edge. Denote by $|\mathcal{B}|$ and $|\mathcal{W}|$ the number of black and white faces, respectively.

Theorem 3.2 Suppose $\mathcal{P}$ is an ideal $\pi / 2$-equiangular polyhedron with $N$ vertices. If $\mathcal{P}$ is 2 -colored with $|\mathcal{B}| \geq|\mathcal{W}|$, then

$$
(N-|\mathcal{W}|) \cdot \frac{V_{8}}{2} \leq \operatorname{vol}(\mathcal{P}) .
$$

This inequality is sharp for an infinite family of polyhedra obtained by gluing together regular ideal hyperbolic octahedra.

Proof Let $\mathcal{P}$ be an ideal $\pi / 2$-equiangular polyhedron, and consider the orbifold $\mathbb{H}^{3} / \Gamma(\mathcal{P})$, where $\Gamma(\mathcal{P})$ is the reflection group generated by $\mathcal{P}$, as described in the introduction. Denote the generators of $\Gamma(\mathcal{P})$ by the same symbol denoting the face of $\mathcal{P}$ through which it is a reflection. If $A$ is a face of $\mathcal{P}$, let $\Gamma_{A}(\mathcal{P})$ be the group obtained from $\Gamma(\mathcal{P})$ by removing the generator $A$ and all relations involving $A$. This may be thought of as "un-mirroring" the face $A$. Then $\operatorname{CC}\left(\mathbb{H}^{3} / \Gamma_{A}(\mathcal{P})\right)$, the convex core of $\mathbb{H}^{3} / \Gamma_{A}(\mathcal{P})$, is an orbifold with totally geodesic boundary and the same volume as $\mathcal{P}$. Note that the face $A$ is a suborbifold of $\mathbb{H}^{3} / \Gamma(\mathcal{P})$ because all dihedral angles of $\mathcal{P}$ are $\pi / 2$. In general, a face of a polyhedron meeting a dihedral angle not equal to $\pi / 2$ will not be a suborbifold and removing the generators and relations corresponding to that face from the reflection group will not give a totally geodesic boundary because the preimage of that face in $\mathbb{H}^{3}$ will not be a collection of disjoint geodesic planes.

To get the best lower bound on volume from Theorem 3.1, the boundary should be chosen to have the greatest possible area. Given a collection of faces $\mathcal{A}=\bigcup_{i=1}^{M} A_{i}$, denote the group obtained by removing all generators and relations involving the $A_{i}$ by $\Gamma_{\mathcal{A}}(\mathcal{P})$. If no two faces in such a collection $\mathcal{A}$ share an edge, the orbifold $\operatorname{CC}\left(\mathbb{H}^{3} / \Gamma_{\mathcal{A}}(\mathcal{P})\right)$ has totally geodesic boundary.

As described before the statement of the theorem, 2-color the faces of $\mathcal{P}$ black and white so that no two faces of the same color share an edge. Suppose that coloring is chosen so that the number of black faces, $|\mathcal{B}|$, is at least the number of white faces, $|\mathcal{W}|$. This choice will ensure that the sum of the areas of the faces in $\mathcal{W}$ is at least the sum of the areas of the black faces, as will be seen in Lemma 3.3. Then, $\operatorname{CC}\left(\mathbb{H}^{3} / \Gamma_{\mathcal{W}}(\mathcal{P})\right)$ is an orbifold with totally geodesic boundary consisting of the $W_{i}$. Theorem 3.1 applied to this orbifold gives

$$
\operatorname{vol}(\mathcal{P}) \geq \operatorname{area}(\mathcal{W}) \cdot \rho_{3}(0)
$$


The following lemma gives the area of $\mathcal{W}$ and completes the proof of the lower bound.

Lemma 3.3 With $\mathcal{W}$ as above,

$$
\operatorname{area}(\mathcal{W})=2 \pi(N-|\mathcal{W}|)
$$

Proof Consider $D \mathcal{W}$, the double of $\mathcal{W}$ along its boundary. Recall that the orbifold Euler characteristic of a 2 -orbifold $Q$ is

$$
\chi(Q)=\chi\left(X_{Q}\right)-\sum_{i}\left(1-1 / m_{i}\right)
$$

where $X_{Q}$ is the underlying topological space and $Q$ has cone points of orders $m_{i}$ [9]. The faces in $\mathcal{W}$ meet each of the $N$ vertices and each of the $E$ edges of $\mathcal{P}$. The orbifold $D \mathcal{W}$ is a union over all faces of $\mathcal{W}$ of doubled ideal polygons. Each of these doubled ideal polygons is a 2 -sphere with a cone point of order $\infty$ for each vertex of the polygon. Each vertex of $\mathcal{P}$ contributes a cone point to two of these doubled polygons. Therefore

$$
\chi(D \mathcal{W})=|\mathcal{W}| \cdot \chi\left(S^{2}\right)-2 N=2(|\mathcal{W}|-N) .
$$

The punctured surface $D \mathcal{W}$ is hyperbolic, being a union of hyperbolic 2-orbifolds. Therefore, the Gauss-Bonnet theorem for orbifolds implies that

$$
\operatorname{area}(D \mathcal{W})=-2 \pi \chi(D \mathcal{W})=4 \pi(N-|\mathcal{W}|),
$$

so that $\operatorname{area}(\mathcal{W})=2 \pi(N-|\mathcal{W}|)$.

The polyhedra which realize the lower bound as claimed in Theorem 3.2 are constructed by gluing together octahedra. Consider a regular ideal hyperbolic octahedron with faces colored white and black, so that no two faces of the same color share an edge. For a single octahedron, $N=6$ and $|\mathcal{W}|=4$, so the lower bound is equal to the volume. To obtain an infinite number of polyhedra which satisfy the claim, glue a finite collection of 2-colored regular ideal hyperbolic octahedra together, only gluing black faces to black faces. Each successive gluing results in a polyhedron with 3 more vertices and 1 more white face. Therefore by induction, for each example constructed in this fashion, the lower inequality in Theorem 3.2 will be equality. See Figure 1. Note that gluing octahedra in a different pattern than described yields examples which do not satisfy the claim. This completes the proof of Theorem 3.2.

The argument giving the lower bound in Theorem 2.2 as a consequence of Theorem 3.2 is similar to the proof of Theorem 5 in [14]. The idea is to average the estimate coming from the black faces with the estimate coming from the white faces. Consider a sort of 


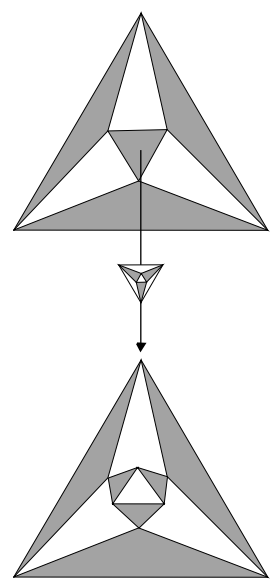

Figure 1: An example of the gluing

dual polyhedron, $\mathcal{G}$, to $\mathcal{P}$. The vertices of $\mathcal{G}$ are the white faces in the specified coloring of $\mathcal{P}$. For any two faces of $\mathcal{P}$ which share a vertex, the corresponding two vertices in $\mathcal{G}$ are connected by an edge. The 2-skeleton is homeomorphic to $S^{2}$, so the Euler characteristic of $\mathcal{G}^{(2)}$ is 2 . The number of vertices, edges and faces of $\mathcal{G}^{(2)}$ respectively are $|\mathcal{W}|, N$ and $|\mathcal{B}|$, where $N$ is the number of vertices of $\mathcal{P}$. Hence $|\mathcal{B}|=2-|\mathcal{W}|+N$. An application of Lemma 3.3 yields $\operatorname{area}(\mathcal{B})=2 \pi(N-|\mathcal{B}|)=2 \pi(|\mathcal{W}|-2)$. Hence

$$
\operatorname{vol}(\mathcal{P}) \geq(|\mathcal{W}|-2) \cdot \frac{V_{8}}{2}
$$

Therefore, combining this inequality with the inequality from Theorem 3.2,

$$
\operatorname{vol}(\mathcal{P}) \geq(N-2) \cdot \frac{V_{8}}{4},
$$

proving the lower bound of Theorem 2.2.

The lower bound in Theorem 2.4, where $\mathcal{P}$ is a $\pi / 2$-equiangular polyhedron with vertices which are either finite or ideal, is proved similarly.

Proposition 3.4 Suppose $\mathcal{P}$ is a $\pi / 2$-equiangular hyperbolic polyhedron with $N_{\infty}$ ideal vertices, $N_{F}$ finite vertices and $|\mathcal{F}|$ faces. Then

$$
\frac{8 N_{\infty}+3 N_{F}-4|\mathcal{F}|}{32} \cdot V_{8} \leq \operatorname{vol}(\mathcal{P}) \text {. }
$$

Proof By the four color theorem, a 4-coloring of the faces of $\mathcal{P}$ may be found $[7 ; 10]$. One of the collections of faces of the same color, say $\mathcal{B}$, has area at least area $(\partial \mathcal{P}) / 4$, where area $(\partial \mathcal{P})$ should be interpreted as the sum of the areas of all the faces of $\mathcal{P}$. 
The area of a hyperbolic $k$-gon with interior angles summing to $S$ is $(k-2) \pi-S$. The sum of the interior angles of a face of $\mathcal{P}$ is $n_{F} \cdot \pi / 2$, where $n_{F}$ is the number of finite vertices of the face. Hence the area of a single face is

$$
\pi\left(n_{\infty}+\frac{n_{F}}{2}-2\right)
$$

where $n_{\infty}$ is the number of ideal vertices of the face. Summing over all faces and using the fact that each finite vertex is a vertex of three faces and each ideal vertex is a vertex of four faces gives

$$
\operatorname{area}(\partial \mathcal{P})=\pi \cdot \frac{8 N_{\infty}+3 N_{F}-4|\mathcal{F}|}{2} .
$$

Then since $\operatorname{area}(\mathcal{B}) \geq \operatorname{area}(\partial \mathcal{P}) / 4$, applying Miyamoto's theorem to $\mathcal{B}$ finishes the proof of the proposition.

The lower bound in Theorem 2.3 follows by setting $N_{\infty}=0$.

\section{Lower volume bound for ideal $\pi / 3$-equiangular polyhe- dra}

In this section the lower bound given in Theorem 2.6 will be proved:

Proposition 4.1 If $\mathcal{P}$ is an ideal $\pi / 3$-equiangular polyhedron with $N>4$ vertices, then

$$
\operatorname{vol}(\mathcal{P})>N \cdot \frac{V_{3}}{3}
$$

Before proving this proposition, a few preliminary results about ideal $\pi / 3$-equiangular polyhedra with more than 4 vertices are needed. Consider a $\pi / 3$-equiangular polyhedron, $\mathcal{P}$, in the upper half-space model for $\mathbb{H}^{3}$ with one vertex placed at the point at infinity. The link of each vertex is Euclidean, so must be an equilateral Euclidean triangle since all dihedral angles are $\pi / 3$. Thus the image of $\mathcal{P}$ under the orthogonal projection to the bounding plane of $\mathbb{H}^{3}$ is an equilateral triangle. This triangle will be referred to as the base triangle. The three vertices adjacent to the vertex at infinity will be called corner vertices.

The following is a corollary of Andreev's theorem for $\pi / 3$-equiangular polyhedra.

Corollary 4.2 If $\mathcal{P}$ is a $\pi / 3$-equiangular polyhedron which has more than 4 vertices, then each face of $\mathcal{P}$ has at least 4 edges. 
Proof Suppose for contradiction that $\mathcal{P}$ has a triangular face, $\Delta_{1}$. Andreev's theorem for $\pi / 3$-equiangular polyhedra (Theorem 2.5) says that the dual graph of $\mathcal{P}^{(1)}$ has no prismatic 3-circuits, so at least two of the edges emanating from $\Delta_{1}$ share a vertex. Hence $\mathcal{P}$ contains two adjacent triangular faces, $\Delta_{1}$ and $\Delta_{2}$. Let $v_{1}$ be the vertex of $\Delta_{1}$ which is not contained in $\Delta_{2}$ and $v_{2}$ the vertex of $\Delta_{2}$ which is not contained in $\Delta_{1}$. Let $e_{1}$ and $e_{2}$ be the edges emanating from $v_{1}$ and $v_{2}$ respectively which are not contained in $\Delta_{1}$ or $\Delta_{2}$. The edges $e_{1}$ and $e_{2}$ are both contained in two common faces. See Figure 2. Therefore by convexity, $e_{1}$ and $e_{2}$ must actually be the same edge, which contradicts the fact that $\mathcal{P}$ has more than 4 vertices.

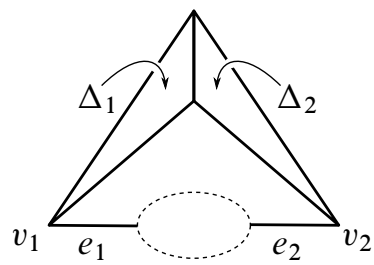

Figure 2: Note that $e_{1}$ and $e_{2}$ are both part of the "front" face and the "back" face.

Lemma 4.3 Suppose that $\mathcal{P}$ is an ideal $\pi / 3$-equiangular polyhedron with $N>4$ vertices. If coordinates for the upper half-space model of $\mathbb{H}^{3}$ are chosen so that a vertex of $\mathcal{P}$ is at the point at infinity, then the Euclidean distance from a corner vertex to each of the adjacent vertices in the base triangle are equal.

Proof The fact that all dihedral angles are equal to $\pi / 3$ implies that the arrangement of defining planes for the corner vertex is left invariant under reflection through a geodesic plane through infinity bisecting the angle between the two vertical planes defining the vertex. This proves the lemma.

The following lemma shows that if $\mathcal{P}$ is a nonobtuse polyhedron, then intersections of faces of $\mathcal{P}$ correspond to intersections of the defining planes of $\mathcal{P}$.

Lemma 4.4 If $\mathcal{P}$ is a nonobtuse hyperbolic polyhedron, then the closures of two faces $F_{1}$ and $F_{2}$ of $\mathcal{P}$ intersect if and only if $\bar{\Pi}_{1}$ and $\bar{\Pi}_{2}$ intersect in $\overline{\mathbb{H}}^{3}$ where $\Pi_{i}$ is the defining plane for $F_{i}$.

Proof Sufficiency is clear.

For necessity, the contrapositive will be proved. Suppose that $F_{1}$ and $F_{2}$ are two faces of $\mathcal{P}$ such that their closures do not intersect. A geodesic orthogonal to both $F_{1}$ 
and $F_{2}$ will be constructed. This geodesic is also orthogonal to both $\Pi_{1}$ and $\Pi_{2}$, and such a geodesic exists only if the closures of the $\Pi_{i}$ are disjoint.

Choose any $x_{0} \in F_{1}$ and $y_{0} \in F_{2}$ and let $\gamma_{0}$ be the geodesic between them. The set

$$
K\left(\gamma_{0}\right)=\left\{(x, y) \in F_{1} \times F_{2} \mid d(x, y) \leq l\left(\gamma_{0}\right)\right\}
$$

is a closed subset of $F_{1} \times F_{2}$. There exists open subsets $N_{i}$ of $F_{i}$ containing all of the ideal vertices of $F_{i}$ such that for any $z_{1} \in N_{1}$ and $z_{2} \in N_{2}, d\left(z_{1}, z_{2}\right)>l\left(\gamma_{0}\right)$. Hence $K\left(\gamma_{0}\right)$ is also a bounded subset of $F_{1} \times F_{2}$, therefore compact.

It follows from compactness of $K\left(\gamma_{0}\right)$ that

$$
d_{\min }=\min \left\{d(x, y) \mid(x, y) \in K\left(\gamma_{0}\right)\right\}
$$

is achieved for some $(x, y) \in K\left(\gamma_{0}\right)$. The geodesic segment, $\gamma$, between $x$ and $y$ must be orthogonal to both $F_{1}$ and $F_{2}$. If not, suppose $\gamma$ is not orthogonal to $F_{1}$. Since $\mathcal{P}$ is nonobtuse, the orthogonal projection of $y$ to $\Pi_{1}$ is contained in $F_{1}$. By the hyperbolic Pythagorean theorem, the geodesic between $y$ and its projection has length less than that of $\gamma$. This contradicts the construction of $\gamma$. The argument is identical if $\gamma$ is not orthogonal to $F_{2}$.

There is actually a simpler argument for the previous lemma in the case that $\mathcal{P}$ is a Coxeter polyhedron. The development of $\mathcal{P}$ into $\mathbb{H}^{3}$ gives a tessellation of $\mathbb{H}^{3}$ by copies of $\mathcal{P}$, so any intersection of defining planes must correspond to an edge of $\mathcal{P}$.

Corollary 4.5 Suppose $\mathcal{P}$ is an ideal $\pi / 3$-equiangular polyhedron with $N>4$ vertices. Choose coordinates for the upper half-space model of $\mathbb{H}^{3}$ so that a vertex, $v_{0}$, of $\mathcal{P}$ is at the point at infinity. Then if the distance in Lemma 4.3 from the corner vertex, $u$, to the two adjacent vertices in the base triangle is $r$ and the edge length of the base triangle is $a$, then $0<r<\frac{3 a}{4}$.

Proof Suppose that the three defining planes which contain $v_{0}$ are $\Pi_{1}, \Pi_{2}$ and $\Pi_{3}$ and that the three defining planes containing $u$ are $\Pi_{1}, \Pi_{2}$ and $\Pi_{4}$. If $\frac{3 a}{4} \leq r<a$, then $\Pi_{3}$ intersects $\Pi_{4}$ with interior dihedral angle less than $\pi / 3$. By Lemma 4.4, the corresponding faces, $F_{3}$ and $F_{4}$, also have intersecting closures, and the interior dihedral angle between $F_{3}$ and $F_{4}$ will be less than $\pi / 3$. If $r=a$, then $\mathcal{P}$ would be a tetrahedron and for $r>a, \mathcal{P}$ would have finite vertices at the points $\Pi_{1} \cap \Pi_{3} \cap \Pi_{4}$ and $\Pi_{2} \cap \Pi_{3} \cap \Pi_{4}$.

In what follows, the intersection with $\mathcal{P}$ of a closed horoball centered at a vertex $u$ of $\mathcal{P}$ which intersects only faces and edges containing $u$ will be called a vertex neighborhood. The next lemma is the main observation which leads to the lower volume bound. This lemma follows the approach of Adams in [1]. 
Lemma 4.6 Let $\mathcal{P}$ be an ideal $\pi / 3$-equiangular polyhedron with more than 4 vertices. Suppose two vertex neighborhoods of equal volume intersect with disjoint interiors. Then the volume of each of the vertex neighborhoods is at least $\sqrt{3} / 6$.

Proof Let $B_{1}$ and $B_{2}$ about vertices $u_{1}$ and $u_{2}$, respectively, be vertex neighborhoods which intersect with disjoint interiors. Choose coordinates for the upper half-space model of $\mathbb{H}^{3}$ so that $u_{1}$ is the point at infinity and $B_{1}$ intersects $B_{2}$ at Euclidean height 1 above the bounding plane. Let $\Gamma(\mathcal{P})$ be the reflection group generated by $\mathcal{P}$ and $\mathcal{G}_{\infty}$ the subgroup fixing the point at infinity:

$$
\mathcal{G}_{\infty}=\{\gamma \in \Gamma(\mathcal{P}) \mid \gamma(\{\infty\})=\{\infty\}\} .
$$

Let $H_{1}=\mathcal{G}_{\infty} \cdot B_{1}$ be the horoball centered at infinity covering $B_{1}$ and let $H_{2}$ be one of the height 1 horoballs contained in $\Gamma(\mathcal{P}) \cdot B_{2}$. The projection of $\mathcal{P}$ to the bounding plane of $\mathbb{H}^{3}$ is an equilateral triangle and the orbit of this triangle under the action of $\mathcal{G}_{\infty}$ tiles the plane. Let $\Delta$ be a triangle in this tiling containing the point of $\overline{\mathbb{H}}^{3}$ about which $\mathrm{H}_{2}$ is centered.

The collection of height 1 horoballs covering $B_{2}$ is equal to $\mathcal{G}_{\infty} \cdot H_{2}$ and, for each pair $g \neq h \in \mathcal{G}_{\infty}$, either $g H_{2} \cap h H_{2}$ is empty, a single point, or $g H_{2}=h H_{2}$. The proof breaks up into three cases. Either $u_{2} \in$ int $\Delta, u_{2}$ is contained in the interior of an edge of $\Delta$, or $u_{2}$ is a vertex of $\Delta$.

If $u_{2} \in$ int $\Delta$, then the projection of $H_{2}$ to the bounding plane must be a closed disk contained in $\Delta$. The minimum possible value of $\operatorname{vol}\left(B_{1}\right)=\operatorname{vol}\left(B_{2}\right)$ occurs when the projection of $\mathrm{H}_{2}$ to the bounding plane is inscribed in $\Delta$ and $\Delta$ has edge length $\sqrt{3}$, as shown in the left of Figure 3. Hence the area of $\Delta$ is $3 \sqrt{3} / 4$ and

$$
\operatorname{vol}\left(B_{i}\right)=\frac{3 \sqrt{3}}{4} \int_{1}^{\infty} \frac{d z}{z^{3}}=\frac{3 \sqrt{3}}{8} .
$$

If $u_{2}$ is contained in the interior of an edge of $\Delta$, then the minimum possible value of the vertex neighborhood volume occurs when $\Delta$ has edge length $2 \sqrt{3} / 3$ and $u_{2}$ is at the midpoint of an edge of $\Delta$, as in the right of Figure 3. Calculating as above,

$$
\operatorname{vol}\left(B_{i}\right)=\frac{\sqrt{3}}{6}
$$

Now suppose that $u_{2}$ is a vertex of $\Delta$. If the edge length of $\Delta$ is $a$, then

$$
\operatorname{vol}\left(B_{1}\right)=\frac{a^{2} \sqrt{3}}{4} \int_{1}^{\infty} \frac{d z}{z^{3}}=\frac{a^{2} \sqrt{3}}{8} .
$$



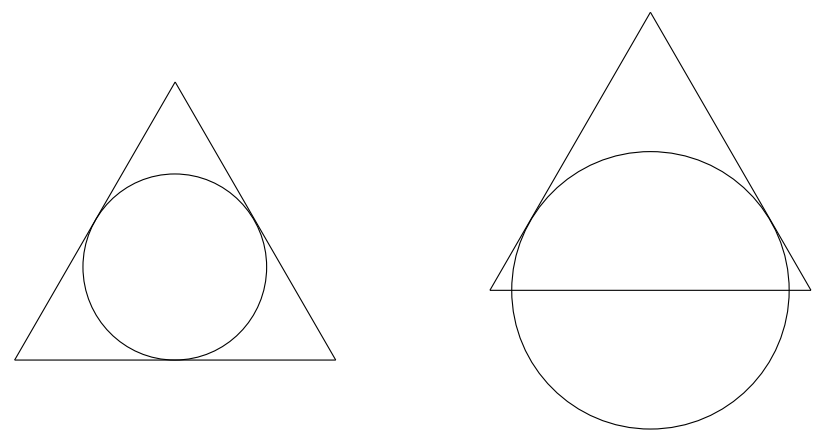

Figure 3: On the left is the projection of $H_{2}$ inscribed in $\Delta$. On the right is the projection of $H_{2}$ in the case where $u_{2}$ is contained in the interior of an edge of $\Delta$.

If $r$ is as in Corollary 4.5, then

$$
\operatorname{vol}\left(B_{2}\right)=\frac{\sqrt{3}}{8 r^{2}}
$$

By Corollary 4.5, $0<r<\frac{3 a}{4}$, so

$$
\operatorname{vol}\left(B_{2}\right)>\frac{2 \sqrt{3}}{9 a^{2}} .
$$

Equating this lower bound with $\operatorname{vol}\left(B_{1}\right)$ yields $a=2 \sqrt{3} / 3$. Therefore we may conclude that

$$
\operatorname{vol}\left(B_{i}\right)>\frac{\sqrt{3}}{6} \text {. }
$$

To complete the proof of Proposition 4.1, start with disjoint, equal volume vertex neighborhoods at each vertex. Expand the vertex neighborhoods so that the volumes remain equal at all time until two of the vertex neighborhoods intersect with disjoint interior intersection. Lemma 4.6 then says that there is a vertex neighborhood at each vertex of volume at least $\sqrt{3} / 6$. Böröczky and Florian in [8] show that the maximal density of a horoball packing in $\mathbb{H}^{3}$ is $\sqrt{3} /\left(2 V_{3}\right)$. Applying this result gives

$$
\operatorname{vol}(\mathcal{P})>N \cdot \frac{V_{3}}{3} \text {. }
$$

In a personal communication, Rivin has indicated how to improve the lower bound to $N \cdot\left(3 V_{3} / 8\right)$. The idea of the argument is that for any given vertex $v$ in a $\pi / 3-$ equiangular polyhedron $\mathcal{P}, v$ along with the three vertices of $\mathcal{P}$ with which $v$ shares an edge are the vertices of a regular ideal hyperbolic tetrahedron contained in $\mathcal{P}$. A collection of such tetrahedra with disjoint interiors may be constructed by taking any 
independent set of vertices of $\mathcal{P}$. By a result of Heckman and Thomas, a trivalent graph with $N$ vertices contains an independent set of cardinality at least $3 N / 8$ [12].

\section{The upper volume bounds}

In this section, the upper volume bounds in Theorems 2.2, 2.3, 2.4 and 2.6 will be proved using arguments inspired by an argument of Agol and D Thurston for an upper bound on the volume of an alternating link complement [14]. First, a decomposition of an arbitrary nonobtuse hyperbolic polyhedron into tetrahedra will be described. In each case, the volume contributed by the tetrahedra meeting at each vertex will be analyzed to obtain the volume bounds.

Let $\mathcal{P}$ be a nonobtuse hyperbolic polyhedron and $v_{0}$ a vertex of $\mathcal{P}$. For each face, $A_{i}$, not containing $v_{0}$, let $\gamma_{i}$ be the unique geodesic orthogonal to $A_{i}$ which passes through or limits to $v_{0}$, where $v_{0}$ is a finite or ideal vertex respectively. Define the nearest point projection, $u_{i}$, of $v_{0}$ to $A_{i}$ to be the intersection of $\gamma_{i}$ with $A_{i}$. The projection $u_{i}$ will lie on the interior of $A_{i}$ unless $A_{i}$ meets one of the faces containing $v_{0}$ orthogonally, in which case, $u_{i}$ will lie in the interior of an edge of $A_{i}$ or will coincide with a vertex of $A_{i}$ if $A_{i}$ meets two faces containing $v_{0}$ orthogonally. Cyclically label the vertices of $A_{i}$ by $v_{i, j}$ where $j \in\left\{1,2, \ldots, \operatorname{deg}\left(A_{i}\right)\right\}$ is taken modulo $\operatorname{deg}\left(A_{i}\right)$. Let $w_{i, j}$ be the nearest point projection of $u_{i}$ onto the edge of $A_{i}$ with endpoints $v_{i, j}$ and $v_{i, j+1}$, where the nearest point projection is defined as above. Each face of a nonobtuse polyhedron is a nonobtuse polygon, so the nearest point projection of any point in $A_{i}$ to an edge $A_{i}$ actually lies in $A_{i}$. See Figure 4.
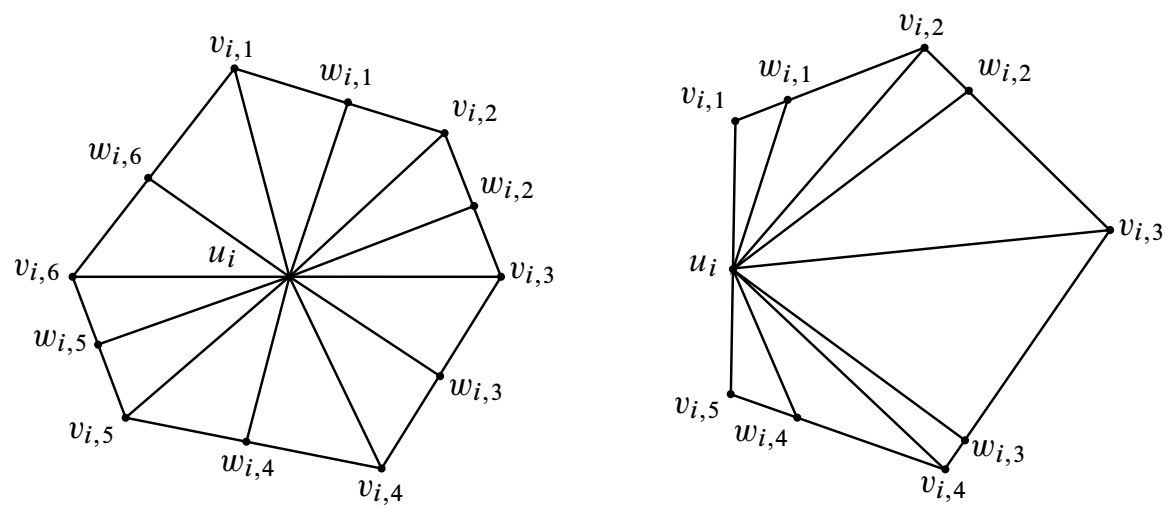

Figure 4: The figure on the left shows the case where $u_{i}$ is in the interior of a face. The figure on the right is the case where $u_{i}$ is in the interior of an edge. 
Define $\Delta(i, j)$ to be the tetrahedron with vertices $v_{0}, u_{i}, w_{i, j}$ and $v_{i, j}$ and $\Delta^{\prime}(i, j)$ to be the tetrahedron with vertices $v_{0}, u_{i}, w_{i, j}$ and $v_{i, j+1}$. In the case where $u_{i}$ coincides with $w_{i, j}$, both $\Delta(i, j)$ and $\Delta^{\prime}(i, j)$ will be degenerate tetrahedra. Let $\mathcal{I}$ be the set of $(i, j)$ such that $\Delta(i, j)$ and $\Delta^{\prime}(i, j)$ are nondegenerate. For each $(i, j) \neq\left(i^{\prime}, j^{\prime}\right) \in \mathcal{I}$, $\operatorname{Int}(\Delta(i, j)) \cap \operatorname{Int}\left(\Delta\left(i^{\prime}, j^{\prime}\right)\right)=\varnothing$ and $\operatorname{Int}\left(\Delta^{\prime}(i, j)\right) \cap \operatorname{Int}\left(\Delta^{\prime}\left(i^{\prime}, j^{\prime}\right)\right)=\varnothing$. Also, the interior of each $\Delta$ is disjoint from the interior of each $\Delta^{\prime}$. Then

$$
\mathcal{P}=\bigcup_{(i, j) \in \mathcal{I}}\left(\Delta(i, j) \cup \Delta^{\prime}(i, j)\right) .
$$

This decomposition of $\mathcal{P}$ into tetrahedra will be analyzed to prove each of the upper bounds in Theorems 2.2, 2.3, 2.4 and 2.6. The following technical lemma is needed. It follows directly from the fact that the Lobachevsky function is concave down on the interval $[0, \pi / 2]$.

Lemma 5.1 Suppose $\vec{\alpha}=\left(\alpha_{1}, \ldots, \alpha_{M}\right)$ where $\alpha_{i} \in[0, \pi / 2]$. Let

$$
f(\vec{\alpha})=\frac{1}{2} \sum_{i=1}^{M} \Lambda\left(\pi / 2-\alpha_{i}\right)
$$

and $g(\vec{\alpha})=\alpha_{1}+\cdots+\alpha_{M}$. Then the maximum value of $f(\vec{\alpha})$ subject to the constraint $g(\vec{\alpha})=C$ for some constant $C \in[0, M \pi / 2]$ occurs for $\vec{\alpha}=(C / M, \ldots, C / M)$

The next proposition gives the upper bound in Theorem 2.2.

Proposition 5.2 If $\mathcal{P}$ is an ideal $\pi / 2$-equiangular polyhedron with $N$ vertices, then

$$
\operatorname{vol}(\mathcal{P}) \leq(N-4) \cdot \frac{V_{8}}{2},
$$

where $V_{8}$ is the volume of the regular ideal hyperbolic octahedron. Equality is achieved when $\mathcal{P}$ is the regular ideal hyperbolic octahedron.

Proof Decompose $\mathcal{P}$ as above. Suppose that $v=v_{i, j}$ is a vertex of $\mathcal{P}$ which is not contained in a face containing $v_{0}$. Then $v$ is contained in exactly eight tetrahedra of the decomposition, say $T_{1}, \ldots, T_{8}$. Suppose that $T_{l}$ coincides with $\Delta(m, n)$ in the decomposition. Then $T_{l}$ is a tetrahedron with 2 ideal vertices, $v_{0}$ and $v$, and two finite vertices, $u_{m}$ and $w_{m, n}$. The dihedral angles along the edges between $v$ and $u_{m}$, between $u_{m}$ and $w_{m, n}$ and between $w_{m, n}$ and $v_{0}$ are all $\pi / 2$. Suppose that the dihedral angle along the edge between $v$ and $v_{0}$ is $\alpha_{l}$. Then the dihedral angles along the remaining two edges are $\pi / 2-\alpha_{l}$. See Figure 5 . 


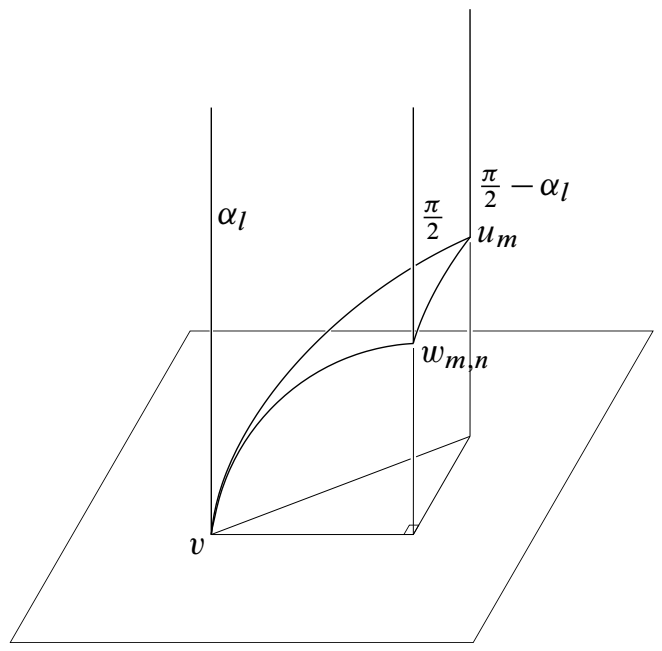

Figure 5: One of the $T_{l}$

The volume of $T_{l}$ is given by $\Lambda\left(\pi / 2-\alpha_{l}\right) / 2$, where the Lobachevsky function, $\Lambda$, is defined as

$$
\Lambda(\theta)=-\int_{0}^{\theta} \log |2 \sin (t)| d t .
$$

Therefore, the volume contributed by the tetrahedra adjacent to the vertex $v$ is a function of $\vec{\alpha}=\left(\alpha_{1}, \alpha_{2}, \ldots, \alpha_{8}\right)$ :

$$
f(\vec{\alpha})=\frac{1}{2} \sum_{l=1}^{8} \Lambda\left(\pi / 2-\alpha_{l}\right) .
$$

The $\alpha_{l}$ must sum to $2 \pi$. The maximum of $f(\vec{\alpha})$, subject to this constraint, occurs when $\vec{\alpha}=(\pi / 4, \pi / 4, \ldots, \pi / 4)$, by Lemma 5.1. Gluing 16 copies of $T_{l}$ with $\alpha_{l}=\pi / 4$ together appropriately yields a regular ideal hyperbolic octahedron. Hence $f(\pi / 4, \pi / 4, \ldots, \pi / 4)=V_{8} / 2$.

Only two tetrahedra in the decomposition meet each of the four vertices which share an edge with $v_{0}$. A similar analysis as above shows that the volume contributed by the tetrahedra at each of these four vertices is no more than $V_{8} / 8$.

Therefore, accounting for the vertex, $v_{0}$, at infinity and the fact that only $V_{8} / 8$ is contributed by each of the tetrahedra at the vertices adjacent to $v_{0}$,

$$
\operatorname{vol}(\mathcal{P}) \leq(N-1) \cdot \frac{V_{8}}{2}-4 \cdot 3 \frac{V_{8}}{8}=(N-4) \cdot \frac{V_{8}}{2} .
$$

Equality is clearly achieved when $\mathcal{P}$ is the regular ideal hyperbolic octahedron. 
The proof of the upper bound in Theorem 2.6 is similar to the previous argument.

Proposition 5.3 If $\mathcal{P}$ is an ideal $\pi / 3$-equiangular polyhedron with $N$ vertices, then

$$
\operatorname{vol}(\mathcal{P}) \leq(3 N-14) \cdot \frac{V_{3}}{2},
$$

where $V_{3}$ is the volume of the regular ideal hyperbolic tetrahedron. Equality is achieved when $\mathcal{P}$ is the regular ideal hyperbolic cube.

Proof Decompose $\mathcal{P}$ as described at the beginning of this section. Each vertex of $\mathcal{P}$ which is not contained in a face containing $v_{0}$ is a vertex of exactly six tetrahedra of the decomposition. Lemma 5.1 implies that the sum of the volumes of the six tetrahedra around such a vertex is no more than $3 V_{3} / 2$.

If $v_{1}$ is one of the three vertices adjacent to $v_{0}$, then $v$ is a vertex of two tetrahedra, $T_{1}$ and $T_{2}$, say. The sum of the volumes of $T_{1}$ and $T_{2}$ is at most $V_{3} / 3$ when $\alpha_{1}=\alpha_{2}=\pi / 6$, again by Lemma 5.1.

By Corollary 4.2 each face containing $v_{0}$ has degree at least 4 . If $v_{2}$ is a vertex of such a face which does not share an edge with $v_{0}$, then $v_{2}$ is a vertex of four tetrahedra of the decomposition of $\mathcal{P}$. See Figure 6.

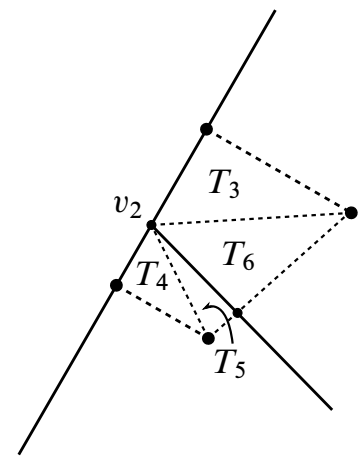

Figure 6: A view of $v_{2}$ as seen from $v_{0}$. The solid lines are edges of $\mathcal{P}$, and the dashed lines are edges of tetrahedra which are not also edges of $\mathcal{P}$.

The link of $v_{2}$ intersected with each of $T_{i}, i=3,4$ is a Euclidean triangle with angles $\pi / 2, \pi / 3$ and $\alpha_{i}$. Hence $\alpha_{3}=\alpha_{4}=\pi / 6$. Using Lemma 5.1 and the fact that $\alpha_{5}+\alpha_{6}=2 \pi / 3$, the sum of the volumes of these four tetrahedra is seen to have a maximum value of $5 V_{3} / 6$.

The upper bound is computed by assuming that the volume contributed by the tetrahedra containing each vertex other than $v_{0}$ is $3 V_{3} / 2$ and subtracting the excess for each of 
the three vertices which share an edge with $v_{0}$ and for the three vertices described in the previous paragraph:

$$
\operatorname{Vol}(\mathcal{P}) \leq\left((N-1) \frac{3}{2}-3\left(\frac{7}{6}\right)-3\left(\frac{2}{3}\right)\right) \cdot V_{3}=\left(\frac{3 N-14}{3}\right) \cdot \frac{3 V_{3}}{2} .
$$

The regular ideal hyperbolic cube has $N=8$ and volume $5 V_{3}$.

The proofs of Theorem 2.3 and Theorem 2.4 require different methods than the previous two theorems because Lemma 5.1 does not apply. The volume of the tetrahedra into which $\mathcal{P}$ is decomposed is given by the sum of three Lobachevsky functions, so the simple Lagrange multiplier analysis fails. The next lemma will play the role of Lemma 5.1 in what follows.

Lemma 5.4 The regular ideal hyperbolic cube has largest volume among all ideal polyhedra with the same combinatorial type.

Proof Any ideal polyhedron, $\mathcal{Q}$, with the combinatorial type of the cube can be decomposed into five ideal tetrahedra as follows: Let $v_{1}, v_{2}, v_{3}, v_{4}$ be a collection of vertices of $\mathcal{Q}$ so that no two share an edge. The five ideal tetrahedra consist of the tetrahedron with vertices $v_{1}, v_{2}, v_{3}$ and $v_{4}$, and the four tetrahedra with vertices consisting of $v_{i}$ along with the three adjacent vertices, for $i=1,2,3,4$.

Then since the regular ideal tetrahedron is the ideal tetrahedron of maximal volume, $\operatorname{vol}(\mathcal{Q}) \leq 5 V_{3}$. The regular ideal hyperbolic cube is decomposed into five copies of the regular ideal tetrahedron when the above decomposition is applied, so has volume $5 V_{3}$.

The next proposition proves the upper bound in Theorem 2.3.

Proposition 5.5 If $\mathcal{P}$ is a $\pi / 2$-equiangular compact hyperbolic polyhedron with $N$ vertices, then

$$
\operatorname{vol}(\mathcal{P})<(N-10) \cdot \frac{5 V_{3}}{8} .
$$

Proof Decompose $\mathcal{P}$ into tetrahedra as described at the beginning of this section for some choice of $v_{0}$. The volume of $\mathcal{P}$ will be bounded above by considering tetrahedra with one ideal vertex. The reason for using tetrahedra with an ideal vertex to estimate the volume of a compact polyhedron is that

$$
\max _{v \in \operatorname{Vert}(\mathcal{P})} d\left(v_{0}, v\right)
$$


can be made arbitrarily large by choosing polyhedra $\mathcal{P}$ with a large enough number of vertices.

Suppose that $v=v_{i, j}$ is a vertex of $\mathcal{P}$ which is not contained in a face containing $v_{0}$. The vertex $v$ is contained in six tetrahedra of the decomposition. Consider $S$, the union of the six triangular faces of these tetrahedra which are contained in the faces of the polyhedron which contain $v$. Let $\hat{v}$ be the point at infinity determined by the geodesic ray emanating from $v$ and passing through $v_{0}$. Define $\mathcal{T}$ to be the cone of $S$ to $\widehat{v}$.

The cone, $\mathcal{T}$, is an octant of an ideal cube, $\mathcal{Q}$. By Lemma 5.4 , $\operatorname{vol}(\mathcal{Q}) \leq 5 V_{3}$. Then since $\operatorname{vol}(\mathcal{Q})=8 \operatorname{vol}(\mathcal{T}), \operatorname{vol}(\mathcal{T}) \leq 5 V_{3} / 8$.

By Andreev's theorem, each face of $\mathcal{P}$ must be of degree at least 5 . Hence, the three faces of $\mathcal{P}$ containing $v_{0}$ contain at least 10 distinct vertices of $\mathcal{P}$, so there are at most $N-10$ vertices that do not share a face with $v_{0}$. Therefore the volume of $\mathcal{P}$ satisfies

$$
\operatorname{vol}(\mathcal{P})<(N-10) \cdot \frac{5 V_{3}}{8} .
$$

Proposition 5.6 combines the techniques of Proposition 5.2 and Proposition 5.5 and gives the upper bound in Theorem 2.4.

Proposition 5.6 If $\mathcal{P}$ is a $\pi / 2$-equiangular hyperbolic polyhedron with $N_{\infty} \geq 1$ ideal vertices and $N_{F}$ finite vertices, then

$$
\operatorname{vol}(\mathcal{P})<\left(\mathrm{N}_{\infty}-1\right) \cdot \frac{V_{8}}{2}+N_{F} \cdot \frac{5 V_{3}}{8} .
$$

Proof Assign to one of the ideal vertices the role of $v_{0}$ in the decomposition described at the beginning of this section. Then each ideal vertex of $\mathcal{P}$ which is not contained in a face of $\mathcal{P}$ containing $v_{0}$ will be a vertex of eight tetrahedra in the decomposition. These tetrahedra contribute no more than $V_{8} / 2$ to the volume of $\mathcal{P}$, by Proposition 5.2. Each finite vertex which is not contained in a face containing $v_{0}$ is a vertex of six tetrahedra. The volume contributed by these is no more than $5 V_{3} / 8$ by Lemma 5.4 and the proof of Proposition 5.5. Putting this all together completes the proof.

\section{Sequences of polyhedra that realize the upper bound esti- mates}

In this section, it is proved that the upper bounds in Theorems 2.2, 2.3 and 2.6 are asymptotically sharp. Results will first be established about the convergence of sequences of 
circle patterns in the plane and about the convergence of volumes of polyhedra which correspond to these circle patterns.

Define a disk pattern to be a collection of closed round disks in the plane such that no disk is the Hausdorff limit of a sequence of distinct disks and so that the boundary of any disk is not contained in the union of two other disks. Define the angle between two disks to be the angle between a clockwise tangent vector to the boundary of one disk at an intersection point of their boundaries and a counterclockwise tangent vector to the boundary of the other disk at the same point. Suppose that $D$ is a disk pattern such that for any two intersecting disks, the angle between them is in the interval $[0, \pi / 2]$. Define $G(D)$ to be the graph with a vertex for each disk and an edge between any two vertices whose corresponding disks have nonempty interior intersection. The graph $G(D)$ inherits an embedding in the plane from the disk pattern. Identify $G(D)$ with its embedding. A face of $G(D)$ is a component of the complement of $G(D)$. Label the edges of $G(D)$ with the angles between the intersecting disks. The graph $G(D)$ along with its edge labels will be referred to as the labeled 1-skeleton for the disk pattern $D$. A disk pattern $D$ is said to be rigid if $G(D)$ has only triangular and quadrilateral faces and each quadrilateral face has the property that the four corresponding disks of the disk pattern intersect in exactly one point. See He [11] for more details on disk patterns.

Consider the path metric on $G(D)$ obtained by giving each edge of $G(D)$ length 1 . Given a disk $d$ in a disk pattern $D$, the set of disks corresponding to the ball of radius $n$ in $G(D)$ centered at the vertex corresponding to $d$ will be referred to as $n$ generations of the pattern about $d$. Given disk patterns $D$ and $D^{\prime}$ and disks $d \in D$ and $d^{\prime} \in D^{\prime}$, then $(D, d)$ and $\left(D^{\prime}, d^{\prime}\right)$ agree to generation $n$ if there is a label preserving graph isomorphism between the balls of radius $n$ centered at the vertices corresponding to $d$ and $d^{\prime}$. The following proposition is a slight generalization of the Hexagonal Packing Lemma in [16].

Proposition 6.1 Let $c_{\infty}$ be a disk in an infinite rigid disk pattern $D_{\infty}$. For each positive integer $n$, let $D_{n}$ be a rigid finite disk pattern containing a disk $c_{n}$ so that $\left(D_{\infty}, c_{\infty}\right)$ and $\left(D_{n}, c_{n}\right)$ agree to generation $n$. Then there exists a sequence $s_{n}$ decreasing to 0 such that the ratios of the radii of any two disks adjacent to $c_{n}$ differ from 1 by less than $s_{n}$.

Proof With Lemma 7.1 from [11] playing the role of the ring lemma in [16], the proof runs exactly the same. The length-area lemma generalizes to this case with no change and any reference to the uniqueness of the hexagonal packing in the plane should be replaced with Rigidity Theorem 1.1 from [11]. 
A simply connected disk pattern is a disk pattern so that the union of the disks is simply connected. Disk patterns arising from finite volume hyperbolic polyhedra will all be simply connected, so all disk patterns will be implicitly assumed to be simply connected. If for a simply connected disk pattern $D$, all labels on $G(D)$ are in the interval $(0, \pi / 2]$, Andreev's theorem implies that each face of $G(D)$ will be a triangle or quadrilateral. An ideal disk pattern, $D$, is one where the labels of $G(D)$ are in the interval $(0, \pi / 2]$ and the labels around each triangle or quadrilateral in $G(D)$ sum to $\pi$ or $2 \pi$ respectively. Ideal disk patterns correspond to ideal polyhedra. A nonideal disk pattern, $D$, is one where $G(D)$ has only triangular faces and the sum of the labels around each face is greater than $\pi$. These disk patterns correspond to compact polyhedra.

Ideal disk patterns and their associated polyhedra will be dealt with first. The analysis for nonideal disk patterns is slightly different and will be deferred until after the proofs of the remaining claims in Theorem 2.2 and Theorem 2.6. The upper half-space model for $\mathbb{H}^{3}$ will be used here. For each disk $d$ in the circle pattern, let $S(d)$ be the geodesic hyperbolic plane in $\mathbb{H}^{3}$ bounded by the boundary of $d$.

Suppose $c$ is a disk in $D$ which intersects $l$ neighboring disks, $d_{1}, \ldots, d_{l}$. In the case of an ideal disk pattern, the intersection of $S(c)$ with each of the $S\left(d_{i}\right)$ is a hyperbolic geodesic. These $l$ geodesics bound an ideal polygon, $p(c) \subset \mathbb{H}^{3}$. If necessary, choose coordinates so that the point at infinity is not contained in $c$. Cone $p(c)$ to the point at infinity and denote the ideal polyhedron thus obtained by $C(p(c))$.

Lemma 6.2 Suppose that $D_{n}$ and $D_{\infty}$ are simply connected, ideal, rigid, disk patterns such that $\left(D_{n}, c_{n}\right)$ and $\left(D_{\infty}, c_{\infty}\right)$ satisfy Proposition 6.1. Then

$$
\lim _{n \rightarrow \infty} \operatorname{vol}\left(C\left(p\left(c_{n}\right)\right)\right)=\operatorname{vol}\left(C\left(p\left(c_{\infty}\right)\right)\right)
$$

where $C\left(p\left(c_{\infty}\right)\right)$ is the cone on the polygon determined by the disk $c_{\infty}$. Moreover, there exists a bounded sequence $0 \leq \epsilon_{n} \leq K<\infty$ converging to zero such that $\left|\operatorname{vol}\left(C\left(p\left(c_{n}\right)\right)\right)-\operatorname{vol}\left(C\left(p\left(c_{\infty}\right)\right)\right)\right| \leq \epsilon_{n}$.

Proof Suppose the dihedral angle between $S\left(c_{n}\right)$ and the vertical face which is a cone on the intersection of $S\left(c_{n}\right)$ and $S\left(d_{i, n}\right)$ is $\alpha_{i}^{n}$ and that the corresponding dihedral angles in $C\left(p\left(c_{\infty}\right)\right)$ are $\alpha_{i}^{\infty}$. Then by Chapter 7 of [19],

$$
\operatorname{vol}\left(C\left(p\left(c_{n}\right)\right)\right)=\sum_{i=1}^{l} \Lambda\left(\alpha_{i}^{n}\right),
$$

where $p\left(c_{n}\right)$ has degree $l$. For each $i, \alpha_{i}^{n}$ converges to $\alpha_{i}^{\infty}$ because $\alpha_{i}^{n}$ is a continuous function of the angle between $c_{n}$ and $d_{i, n}$ and the radii of the two disks, which converge 
to the radii of the corresponding disks in the infinite packing by Proposition 6.1. The function $\Lambda$ is continuous, so convergence of the $\alpha$ 's implies the first statement of the lemma. The second statement is a consequence of the first and the fact that $\operatorname{vol}\left(C\left(p\left(c_{n}\right)\right)\right)$ is finite for all $n$ including $\infty$.

The remaining claims in Theorem 2.2 and Theorem 2.6 can now be proved. First the following proposition is proved:

Proposition 6.3 There exists a sequence of ideal $\pi / 2$-equiangular polyhedra $\mathcal{P}_{i}$ with $N_{i}$ vertices such that

$$
\lim _{i \rightarrow \infty} \frac{\operatorname{vol}\left(\mathcal{P}_{i}\right)}{N_{i}}=\frac{V_{8}}{2}
$$

Proof Let $D_{\infty}$ be the infinite disk pattern defined as

$$
D_{\infty}=\bigcup d_{(p, q)},
$$

where the union ranges over all $(p, q) \in \mathbb{Z}^{2}$ such that both $p$ and $q$ are even or both $p$ and $q$ are odd, and $d_{(p, q)}$ is the disk of radius 1 centered at the point $(p, q)$. Consider the ideal hyperbolic polyhedron with infinitely many vertices, $\mathcal{P}_{\infty}$, corresponding to $D_{\infty}$. This polyhedron has all dihedral angles equal to $\pi / 2$. Applying the decomposition into tetrahedra described in the proof of Proposition 5.2, it is seen that the sum of the volumes of the tetrahedra meeting each vertex is exactly $V_{8} / 2$. A sequence of polyhedra, $\mathcal{P}_{2 k}$, which have volume-to-vertex ratio converging to that of $\mathcal{P}_{\infty}$ will be constructed.

For each even natural number $2 k, k \geq 3$, consider the set of lines in the plane $L_{2 k}=\left\{(x, y) \in \mathbb{R}^{2} \mid y=0, y=2 k\right.$, or $\left.y= \pm x+z, z \in \mathbb{Z}\right\}$. Now let $\mathcal{P}_{2 k}$ be the hyperbolic polyhedron with 1 -skeleton given by

$$
\mathcal{P}_{2 k}^{(1)}=\left\{(x, y) \in L_{2 k} \mid 0 \leq y \leq 2 k\right\} /\{(x, y) \sim(x+2 k, y)\}
$$

and all right angles. See Figure 7 for an illustration of $\mathcal{P}_{6}$. The existence of such a hyperbolic polyhedron is guaranteed by Andreev's theorem. Equivalently, there is a simply connected rigid disk pattern, $D_{2 k}$, in the plane with each disk corresponding to a face and right angles between disks which correspond to intersecting faces. The vertices and faces of $\mathcal{P}_{2 k}$ will be referred to in terms of the $(x, y)$ coordinates of the corresponding vertices and faces of $L_{2 k}$.

The polyhedra $\mathcal{P}_{2 k}$ will prove the proposition. The volume of $\mathcal{P}_{2 k}$ is expressed as the sum of volumes of cones on faces and Lemma 6.2 is used to analyze the limiting volume-to-vertex ratio. 
Choose coordinates for the upper half-space model of $\mathbb{H}^{3}$ so that the vertex $(0,0)$ of $\mathcal{P}_{2 k}$ is located at infinity. Then the volume of $\mathcal{P}_{2 k}$ may be written

$$
\operatorname{vol}\left(\mathcal{P}_{2 k}\right)=\sum_{d} \operatorname{vol}(C(p(d))),
$$

where the sum is taken over all faces $d$ which do not meet the vertex $(0,0)$. Using Lemma 6.2, the volume of each $C(p(d))$ can be estimated in terms of the number of generations of disks surrounding $d$ which agree with $D_{\infty}$.

Fix a disk $d_{\infty} \in D_{\infty}$. For each $m \in \mathbb{Z}, m \geq 0$, define $F_{m}$ to be the set of disks $d \in D_{2 k}$ for which $\left(D_{2 k}, d\right)$ and $\left(D_{\infty}, d_{\infty}\right)$ agree to generation $m$, but do not agree to generation $m+1$. The $2 k$ faces of $\mathcal{P}_{2 k}$ centered at the points $(i+1 / 2, k)$, $0 \leq i \leq 2 k-1$ as well as the $4 k$ faces which share an edge with them except for the face centered at $(0, k-1 / 2)$ make up $F_{k-1}$. Thus

$$
\left|F_{k-1}\right|=6 k-1 \text {. }
$$

The set $F_{k-2}$ consists of the face centered at $(0, k-1 / 2)$ along with the faces centered at the $8 k-1$ points with coordinates $(i+1 / 2, k \pm 1)$ and $(i, k \pm 3 / 2)$ for $0 \leq i \leq 2 k-1$, excluding the face centered at $(0, k-3 / 2)$. In general, for $2 \leq l \leq k-1, F_{k-l}$ consists of the face centered at $(0, k-(2(l-1)-1) / 2)$ along with the faces centered at the $8 k-1$ points with coordinates $(i+1 / 2, k \pm(l-1))$ and $(i, k \pm(2 l-1) / 2)$ for $0 \leq i \leq 2 k-1$, excluding the face centered at $(0, k-(2 l-1) / 2)$. Hence for $2 \leq l \leq k-1$,

$$
\left|F_{k-l}\right|=8 k \text {. }
$$

See Figure 7 for an example.

The polyhedron $\mathcal{P}_{2 k}$ has $8 k^{2}+2 k+2$ faces. In the previous paragraph, it was found that

$$
\left|\bigcup_{l=1}^{k-1} F_{l}\right|=8 k^{2}-10 k-1
$$

The remaining $12 k+3$ faces consist of the following: 4 vertical faces which do not contribute to the volume, one $2 k$-gon, $4 k-2$ triangular faces and $8 k$ rectangular faces in $F_{0}$. The maximum value of the Lobachevsky function, $\Lambda(\theta)$, is attained for $\theta=\pi / 6$ [19]. Hence, the formula for the volume of a cone on an ideal polygon given in the proof of Lemma 6.2 implies that the volume of the cone on the $2 k$-gon is less than or equal to $2 k \Lambda(\pi / 6)$. Similarly, each of the remaining triangular and rectangular faces have volume less than or equal to $4 \Lambda(\pi / 6)$. This implies that the leftover faces have cone volume summing to a value $L \leq 14 k \Lambda(\pi / 6)$. 


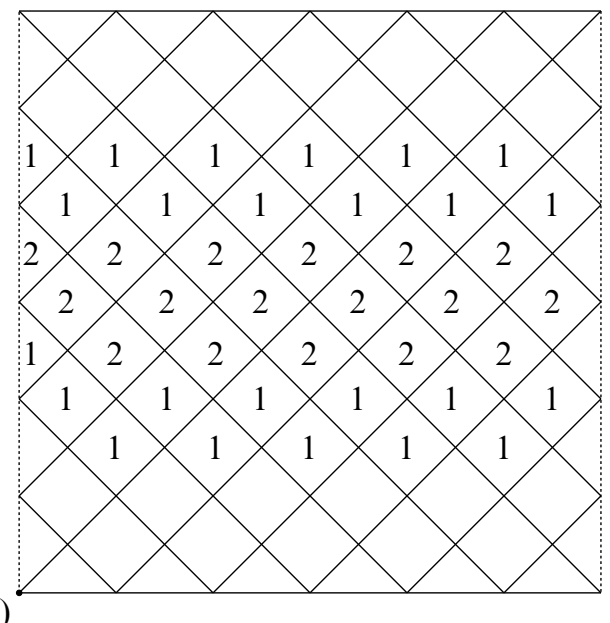

Figure 7: Identify the two vertical sides to obtain $\mathcal{P}_{6}$. A face labeled by an integer $n$ has $n$ generations of disks about it. Unlabeled faces have 0 generations about them.

The volume of the cone to infinity of any face in $D_{\infty}$ is $4 \Lambda(\pi / 4)=V_{8} / 2$. By Lemma 6.2, there exists a real-valued, positive function, $\delta_{m}$, on $F_{m}$ such that for each face $f \in F_{m}, 0 \leq \delta_{m}(f) \leq \epsilon_{m}$ and $\operatorname{vol}(C(f))=V_{8} / 2 \pm \delta_{m}(f)$. Therefore, the volume of $\mathcal{P}_{2 k}$ can written as

$$
\operatorname{vol}\left(\mathcal{P}_{2 k}\right)=\sum_{l=1}^{k-1} \sum_{f \in F_{l}}\left(\frac{V_{8}}{2} \pm \delta_{l}(f)\right)+L .
$$

Using the analysis of the $F_{m}$ from above, expand the sums and collect terms to get

$$
\operatorname{vol}\left(\mathcal{P}_{2 k}\right)=\left(8 k^{2}-10 k-1\right) \frac{V_{8}}{2}+\sum_{l=1}^{k-1} \sum_{f \in F_{l}}\left( \pm \delta_{l}(f)\right)+L
$$

The polyhedron $\mathcal{P}_{2 k}$ has $N_{2 k}=8 k^{2}+2 k$ vertices. Therefore

$$
\lim _{k \rightarrow \infty} \frac{8 k^{2}-10 k-1}{N_{2 k}} \frac{V_{8}}{2}=\frac{V_{8}}{2} .
$$

It remains to show that the ratio of the last two summands to the number of vertices converges to zero. Set $\bar{\delta}_{l}=\max _{f \in F_{l}} \delta_{l}(f)$. Then

$$
\lim _{k \rightarrow \infty} \frac{\left|\sum_{l=1}^{k-1} \sum_{f \in F_{l}}\left( \pm \delta_{l}(f)\right)\right|}{N_{2 k}} \leq \lim _{k \rightarrow \infty}\left(\frac{(6 k-1) \bar{\delta}_{k-1}}{N_{2 k}}+\frac{8 k \sum_{l=1}^{k-2} \bar{\delta}_{l}}{N_{2 k}}\right)=0
$$


because $\bar{\delta}_{l} \rightarrow 0$ as $l \rightarrow \infty$. Also since $L<14 k \Lambda(\pi / 6)$,

$$
\lim _{k \rightarrow \infty} \frac{L}{N_{2 k}}=0
$$

Therefore,

$$
\lim _{k \rightarrow \infty} \frac{\operatorname{vol}\left(\mathcal{P}_{2 k}\right)}{N_{2 k}}=\frac{V_{8}}{2}
$$

The argument to prove Proposition 6.3 easily adapts to prove the following:

Proposition 6.4 There exists a sequence of ideal $\pi / 3$-equiangular polyhedra $\mathcal{P}_{i}$ with $N_{i}$ vertices such that

$$
\lim _{i \rightarrow \infty} \frac{\operatorname{vol}\left(\mathcal{P}_{i}\right)}{N_{i}}=\frac{3 V_{3}}{2}
$$

Proof Consider the regular hexagon $H$ in the plane formed by the vertices $(0,0)$, $(1,0),(3 / 2, \sqrt{3} / 2),(1, \sqrt{3}),(0, \sqrt{3})$ and $(-1 / 2, \sqrt{3} / 2)$. Let $G$ be the lattice of translations generated by $\{(x, y) \mapsto(x+3, y)\}$ and $\{(x, y) \mapsto(x+3 / 2, y+\sqrt{3} / 2)$. Now define $T$ to be the orbit of the hexagon $H$ under the action of $G$ on the plane. This orbit is a tiling of the plane by regular hexagons. As in the previous construction, define

$$
L_{2 k}=\left\{(x, y) \in \mathbb{R}^{2} \mid y=0, y=2 k \sqrt{3} \text {, or }(x, y) \text { lies on a vertex or edge of } T\right\} .
$$

Let $\mathcal{Q}_{2 k}$ be the hyperbolic polyhedron with 1 -skeleton

$$
\mathcal{Q}_{2 k}^{(1)}=\left\{(x, y) \in L_{2 k} \mid 0 \leq y \leq 2 k \sqrt{3}\right\} /\{(x, y) \sim(x+3 k, y)\}
$$

and all dihedral angles $\pi / 3$. This is a polyhedron with $4 k^{2}+k+2$ faces and $N_{2 k}=8 k^{2}+2 k$ vertices. Let $D_{2 k}$ be the associated simply connected rigid disk pattern and $D_{\infty}$ to be the infinite circle pattern with $G\left(D_{\infty}\right)$ equal to a tiling of the plane by equilateral triangles with each edge labeled $\pi / 3$.

As in the proof of Proposition 6.3, choose coordinates so that the vertex at $(0,0)$ is at infinity. Recall that for a fixed choice of $d_{\infty} \in D_{\infty}, F_{m}$ is defined to be the set of disks $d \in D_{2 k}$ for which $\left(D_{2 k}, d\right)$ and $\left(D_{\infty}, d_{\infty}\right)$ agree to generation $m$, but do not agree to generation $m+1$. The set $F_{k-1}$ consists of $3 k-1$ faces, while the remaining $F_{k-l}$ for $2 \leq l \leq k-1$ consist of $4 k$ faces. Again, the faces not contained in $F_{k-l}$ for $1 \leq l \leq k-1$ have cone volume summing to a value $L$ bounded above by a constant multiple of $k$ where the bound is independent of $k$. The volume of the cone to infinity 


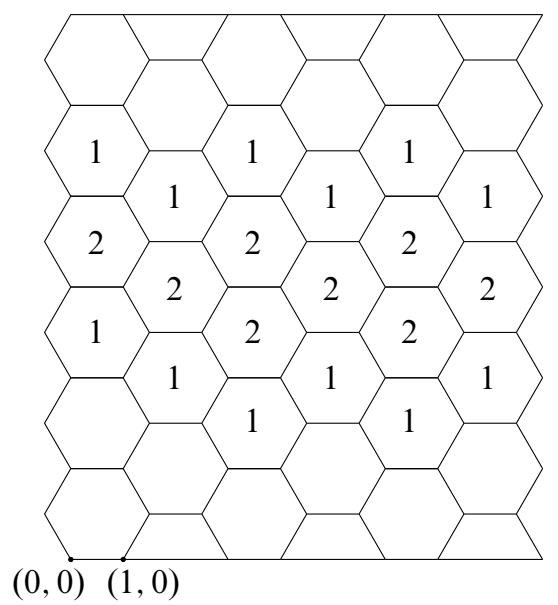

Figure 8: Identify the left and right sides to get $\mathcal{Q}_{6}$. The labeling is as in the previous figure.

of any face in the regular hexagonal circle pattern is $6 \Lambda(\pi / 6)=3 V_{3}$. There exists a function, $\delta_{l}$, with the same properties as above. The volume of $\mathcal{Q}_{2 k}$ is

$$
\operatorname{vol}\left(\mathcal{Q}_{2 k}\right)=\sum_{l=1}^{k-1} \sum_{f \in F_{l}}\left(3 V_{3} \pm \delta_{l}(f)\right)+L .
$$

The argument finishes exactly as the all right-angled case to give

$$
\lim _{k \rightarrow \infty} \frac{\operatorname{vol}\left(\mathcal{Q}_{2 k}\right)}{N_{2 k}}=\frac{3 V_{3}}{2} .
$$

To finish the proof of Theorem 2.3, a minor modification to Lemma 6.2 is made.

Recall that for a disk pattern $D, G(D)$ is the graph with a vertex for each disk and an edge connecting two vertices which have corresponding disks with nonempty interior intersection. Suppose that $D$ is a nonideal disk pattern and that $c$ is a disk which intersects $l$ neighboring disks, $d_{1}, \ldots, d_{l}$. The intersection of $S(c)$ with each of the $S\left(d_{i}\right)$ is a finite length geodesic segment. The union of the $l$ geodesic segments along with the disk bounded by them in $S(c)$ is a polygon $p(c)$. Let $x_{0} \in \mathbb{H}^{3}$ be a point which is not contained in $S(c)$. Denote by $C\left(p(c), x_{0}\right)$ the cone of $p(c)$ to the point $x_{0}$. The cone to the point at infinity in the upper half-space model of $\mathbb{H}^{3}$ will be denoted by $C(p(c),\{\infty\})$.

Let $c$ be a disk in a simply connected, nonideal, finite disk pattern, $D$, with associated polyhedron $\mathcal{P}$. Suppose $c_{\max }$ realizes the quantity

$$
\max _{c^{\prime}} d_{G(D)}\left(c, c^{\prime}\right)
$$


Define a cut point for $c$ to be any vertex of $p\left(c_{\max }\right)$.

Lemma 6.5 Suppose that $D_{n}$ and $D_{\infty}$ are simply connected, nonideal, rigid, disk patterns, such that $\left(D_{n}, c_{n}\right)$ and $\left(D_{\infty}, c_{\infty}\right)$ satisfy Proposition 6.1 and $D_{\infty}$ fills the entire plane. Suppose also that $x_{n}$ is a cut point for $c_{n}$ in $D_{n}$. Then

$$
\lim _{n \rightarrow \infty} \operatorname{vol}\left(C\left(p\left(c_{n}\right), x_{n}\right)\right)=\operatorname{vol}\left(C\left(p\left(c_{\infty}\right),\{\infty\}\right)\right) .
$$

Moreover, there exists a bounded sequence $0 \leq \epsilon_{n} \leq K<\infty$ converging to zero such that $\left|\operatorname{vol}\left(C\left(p\left(c_{n}\right), x_{n}\right)\right)-\operatorname{vol}\left(C\left(p\left(c_{\infty}\right),\{\infty\}\right)\right)\right| \leq \epsilon_{n}$.

Proof Note that for any choice of points $y_{n} \in S\left(c_{n}\right), d\left(x_{n}, y_{n}\right) \rightarrow \infty$ as $n \rightarrow \infty$. Also, since $D_{\infty}$ fills the entire plane, the distance measured in $G\left(D_{n}\right)$ from $c_{n}$ to a disk $c_{n}^{\prime}$ such that $S\left(c_{n}^{\prime}\right)$ contains $x_{n}$ goes to infinity. Hence as $n$ goes to infinity, $x_{n}$ approaches the point at infinity, so the compact cone $C\left(p\left(c_{n}\right), x_{n}\right)$ approaches the infinite cone $C\left(p\left(c_{\infty}\right),\{\infty\}\right)$. Therefore it suffices to show that the volume of the compact cones approaches that of the infinite cone.

A 3-dimensional hyperbolic orthoscheme is a hyperbolic tetrahedron with a sequence of three edges $v_{0} v_{1}, v_{1} v_{2}$ and $v_{2} v_{3}$ such that $v_{0} v_{1} \perp v_{1} v_{2} \perp v_{2} v_{3}$. See Figure 9. Suppose that the degree of $p\left(c_{n}\right)$ is $a_{n}$. The cone, $C\left(p\left(c_{n}\right), x_{n}\right)$, can be decomposed into $2 a_{n}$ orthoschemes by the procedure described at the beginning of Section 5 .

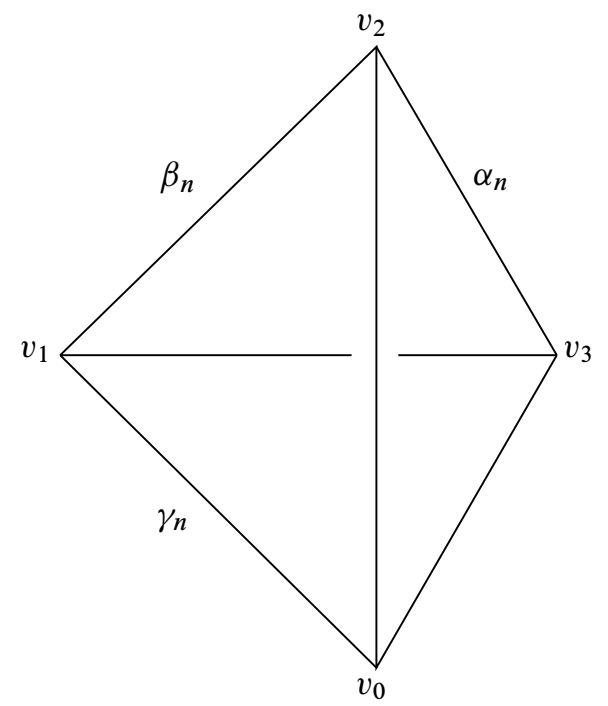

Figure 9: A compact orthoscheme, $T\left(\alpha_{n}, \beta_{n}, \gamma_{n}\right)$. The unlabeled edges have dihedral angle $\pi / 2$. 
The volume of one of the compact orthoschemes, $T\left(\alpha_{n}, \beta_{n}, \gamma_{n}\right)$ determined by angles $\alpha_{n}, \beta_{n}$ and $\gamma_{n}$, as shown in Figure 9, is given by

$$
\begin{aligned}
& \operatorname{vol}\left(T\left(\alpha_{n}, \beta_{n}, \gamma_{n}\right)=\frac{1}{4}\left(\Lambda\left(\alpha_{n}+\delta_{n}\right)-\Lambda\left(\alpha_{n}-\delta_{n}\right)+\Lambda\left(\gamma_{n}+\delta_{n}\right)-\Lambda\left(\gamma_{n}-\delta_{n}\right)\right.\right. \\
& \left.-\Lambda\left(\frac{\pi}{2}-\beta_{n}+\delta_{n}\right)+\Lambda\left(\frac{\pi}{2}-\beta_{n}-\delta_{n}\right)+2 \Lambda\left(\frac{\pi}{2}-\delta_{n}\right)\right), \\
& \text { where } \\
& \text { and } \quad \leq \delta_{n}=\arctan \frac{\sqrt{-\Delta_{n}}}{\cos \alpha_{n} \cos \gamma_{n}}<\frac{\pi}{2}, \\
& \Delta_{n}=\sin ^{2} \alpha_{n} \sin ^{2} \gamma_{n}-\cos ^{2} \beta_{n} .
\end{aligned}
$$

This is due to Lobachevsky. See, for example, page 125 of [4].

Similarly, the cone $C\left(p\left(c_{\infty}\right),\{\infty\}\right)$ can be decomposed into orthoschemes of the form $T\left(\alpha_{\infty}, \pi / 2-\alpha_{\infty}, \gamma_{\infty}\right)$ with one ideal vertex. The volume of this orthoscheme is given by

$$
\operatorname{vol}\left(T\left(\alpha_{\infty}, \pi / 2-\alpha_{\infty}, \gamma_{\infty}\right)=\frac{1}{4}\left(\Lambda\left(\alpha_{\infty}+\gamma_{\infty}\right)+\Lambda\left(\alpha_{\infty}-\gamma_{\infty}\right)+2 \Lambda\left(\pi / 2-\alpha_{\infty}\right)\right) .\right.
$$

As $n \rightarrow \infty, \Delta_{n} \rightarrow-\sin ^{2} \alpha_{n} \cos ^{2} \gamma_{n}$, so $\delta_{n} \rightarrow \alpha_{n}$. Therefore the sequence of volumes of the compact orthoschemes converges to that of the orthoscheme with one ideal vertex. Summing over all orthoschemes in the decomposition proves the lemma.

The next proposition will complete the proof of Theorem 2.3.

Proposition 6.6 There exists a sequence of compact $\pi / 2$-equiangular polyhedra $\mathcal{P}_{i}$ with $N_{i}$ vertices such that

$$
\lim _{i \rightarrow \infty} \frac{\operatorname{vol}\left(\mathcal{P}_{i}\right)}{N_{i}}=\frac{5 V_{3}}{8}
$$

Proof Define $L_{2 k}^{\prime}$ to be $L_{2 k}$ as in the proof of Proposition 6.4 along with the tripods as shown in Figure 10. The tripods must be added to remove the degree 4 faces. Let $\mathcal{R}_{2 k}$ be the polyhedron with $1-$ skeleton

$$
\mathcal{R}_{2 k}^{(1)}=\left\{(x, y) \in L_{2 k}^{\prime} \mid 0 \leq y \leq 2 k \sqrt{3}\right\} /\{(x, y) \sim(x+3 k, y)\}
$$

and all dihedral angles equal to $\pi / 2$. For each $k>2$, this can be realized as a compact hyperbolic polyhedron by Andreev's theorem. The rest of the proof of this proposition mirrors the proof of Proposition 6.4 exactly, using Lemma 6.5 in place of Lemma 6.2. 


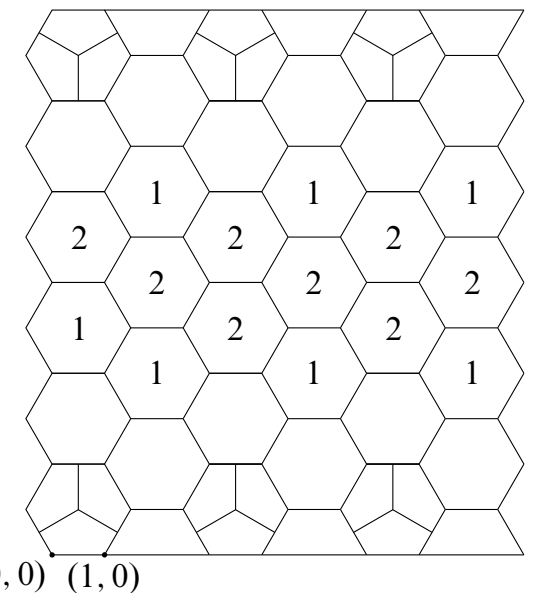

Figure 10: Identify the left and right sides to get $\mathcal{R}_{6}$. The labeling is as in the previous figures.

\section{References}

[1] C C Adams, Volumes of hyperbolic 3-orbifolds with multiple cusps, Indiana Univ. Math. J. 41 (1992) 149-172 MR1160907

[2] I Agol, Finiteness of arithmetic Kleinian reflection groups, from: "International Congress of Mathematicians. Vol. II", Eur. Math. Soc., Zürich (2006) 951-960 MR2275630

[3] I Agol, P A Storm, W P Thurston, Lower bounds on volumes of hyperbolic Haken 3manifolds, J. Amer. Math. Soc. 20 (2007) 1053-1077 MR2328715 With an appendix by $\mathrm{N}$ Dunfield

[4] D V Alekseevskij, È B Vinberg, A S Solodovnikov, Geometry of spaces of constant curvature, from: "Geometry, II”, (R V Gamkrelidze, È B Vinberg, editors), Encyclopaedia Math. Sci. 29, Springer, Berlin (1993) 1-138 MR1254932

[5] E M Andreev, Convex polyhedra in Lobačevskiŭ spaces, Mat. Sb. (N.S.) 81 (123) (1970) 445-478 MR0259734

[6] E M Andreev, Convex polyhedra of finite volume in Lobačevskiŭ space, Mat. Sb. (N.S.) 83 (125) (1970) 256-260 MR0273510

[7] K Appel, W Haken, Every planar map is four colorable, Contemporary Math. 98, Amer. Math. Soc. (1989) MR1025335 With the collaboration of J Koch

[8] K Böröczky, A Florian, Über die dichteste Kugelpackung im hyperbolischen Raum, Acta Math. Acad. Sci. Hungar 15 (1964) 237-245 MR0160155

[9] D Cooper, CD Hodgson, S P Kerckhoff, Three-dimensional orbifolds and conemanifolds, MSJ Memoirs 5, Math. Soc. Japan, Tokyo (2000) MR1778789 With a postface by S Kojima 
[10] G Gonthier, A computer-checked proof of the Four Colour Theorem, Preprint (2005) Available at http://research.microsoft.com/en-us/um/people/gonthier/ 4colproof.pdf

[11] Z-X He, Rigidity of infinite disk patterns, Ann. of Math. (2) 149 (1999) 1-33 MR1680531

[12] C C Heckman, R Thomas, Independent sets in triangle-free cubic planar graphs, J. Combin. Theory Ser. B 96 (2006) 253-275 MR2208354

[13] T Inoue, Organizing volumes of right-angled hyperbolic polyhedra, Algebr. Geom. Topol. 8 (2008) 1523-1565 MR2443253

[14] M Lackenby, The volume of hyperbolic alternating link complements, Proc. London Math. Soc. (3) 88 (2004) 204-224 MR2018964 With an appendix by I Agol and D Thurston

[15] Y Miyamoto, Volumes of hyperbolic manifolds with geodesic boundary, Topology 33 (1994) 613-629 MR1293303

[16] B Rodin, D Sullivan, The convergence of circle packings to the Riemann mapping, J. Differential Geom. 26 (1987) 349-360 MR906396

[17] A Selberg, On discontinuous groups in higher-dimensional symmetric spaces, from: "Contributions to function theory (internat. Colloq. Function Theory, Bombay, 1960)", Tata Inst. of Fund. Research, Bombay (1960) 147-164 MR0130324

[18] E Steinitz, Polyeder und Raumeinteilungen, Enzylk. Math. Wiss. 3 (1922) 1-139

[19] W P Thurston, The geometry and topology of three-manifolds, Princeton Univ. Math. Dept. Lecture Notes (1979) Available at http://msri.org/publications/books/ gt $3 \mathrm{~m} /$

Department of Mathematics, Statistics and Computer Science, University of Illinois at Chicago 851 S Morgan St, Chicago, IL 60607

atkinson@math . uic.edu

http://www.math.uic.edu/ atkinson

Received: 26 June 2008 Revised: 6 May 2009 